\title{
smaug protein represses translation of unlocalized nanos mRNA in the Drosophila embryo
}

\author{
Craig A. Smibert, Joan E. Wilson, Karen Kerr, and Paul M. Macdonald ${ }^{1}$ \\ Department of Biological Sciences, Stanford University, Stanford, California 94305-5020 USA
}

\begin{abstract}
nanos mRNA, which encodes the localized component of the Drosophila posterior body patterning determinant, is normally translated only at the posterior pole of the embryo, where the mRNA is concentrated. Here we identify two similar cis-acting sequences in the nanos mRNA 3' untranslated region that mediate translational repression. These sequences bind an embryonic protein of $135 \mathrm{kD}$, smaug, and we refer to them as smaug recognition elements (SREs). Analysis of point mutations in the SREs reveals a strong correlation between smaug binding and translational repression; mutants unable to bind smaug in vitro are not repressed translationally in vivo, whereas mutants that do bind smaug remain repressed translationally. These results strongly suggest that smaug acts in translational repression of unlocalized nanos mRNA. Translational repression is essential, as embryos expressing a nanos mRNA with mutated SREs develop with anterior body patterning defects and die, despite correct localization of the RNA.
\end{abstract}

[Key Words: nanos; smaug protein; Drosophila embryo; translational regulation]

Received July 3, 1996; revised version accepted August 22, 1996.

Early development is often programmed by maternally provided mRNAs (Davidson 1986). The activities of these mRNAs must be regulated to ensure an orderly progression from one developmental stage to the next. Two mechanisms with prominent roles in controlling the activities of maternal mRNAs are translational regulation and mRNA localization; frequently, these posttranscriptional controls act together to restrict narrowly the expression of a particular mRNA. Striking examples of these phenomena are found in Drosophila where the basic body plan is established in large part by proteins whose distributions are governed by both mRNA localization and translational control (for review, see Curtis et al. 1995).

Posterior body patterning in Drosophila involves the sequential actions of two cytoplasmic determinants encoded by the oskar (osk) and nanos (nos) genes. Both determinants are localized to the posterior of the oocyte or embryo, and this localization is essential; if either protein is expressed ectopically, body patterning is reprogrammed with lethal consequences (Ephrussi and Lehmann 1992; Gavis and Lehmann 1992; Smith et al. 1992; Webster et al. 1994). Localization of each determinant requires prelocalization of the corresponding mRNA. osk mRNA is localized to the posterior pole of the oocyte during mid-oogenesis (Ephrussi et al. 1991; Kim-Ha et al. 1991), whereas nos mRNA becomes concentrated at the

${ }^{1}$ Corresponding author. same site much later in oogenesis (Gavis and Lehmann 1992; Wang et al. 1994). Both mRNAs are also subject to translational regulation (Gavis and Lehmann 1994; KimHa et al. 1995; Markussen et al. 1995; Rongo et al. 1995), as might be expected given the consequences of ectopic osk or nos expression. In principle, translational control could cooperate with mRNA localization to tightly restrict expression of an RNA in two ways: (1) by restraining protein synthesis until localization is complete, and (2) by blocking translation of transcripts that escape the localization process. Both of these roles for translational control require a functional link between localization and translation, a property first established by Gavis and Lehmann (1994), who demonstrated that activation of nos translation requires posterior localization of the nos mRNA. In the case of osk mRNA, where such a link also exists, one demonstrated role for translational repression is in inactivating transiently the mRNA until localization is complete (Kim-Ha et al. 1995). This repression requires regulatory sequences, bruno response elements (BREs), in the osk mRNA 3' untranslated region (UTR), which bind to the bruno RNA-binding protein (Kim-Ha et al. 1995). It is not yet clear whether the other role for translational repression, namely to block expression from mRNA that escapes localization, is used to limit the activity of osk mRNA. Translational repression of nos mRNA has been characterized less extensively, and the factors and precise sequences involved in translational control have not been identified. Moreover, the nature of the link between translation and mRNA local- 
ization is not understood. However, it is known that nos translation is repressed by the nos $3^{\prime}$ UTR, and activation of nos translation requires osk (Gavis and Lehmann 1994).

Here we show that translational regulation of nos mRNA serves specifically to repress translation of nos transcripts that fail to localize to the posterior. Repression triggers degradation, a logical outcome to ensure that this unlocalized mRNA is never translated. We identify and characterize the cis-acting regulatory elements [designated smaug recognition elements (SREs)] that mediate these events. The SREs bind the RNA-binding protein smaug $(\mathrm{smg})$. Failure to bind smg leads to translational derepression, suggesting that smg represses nos translation through the SREs. The ensuing ectopic expression of nos protein is lethal, demonstrating that repression of nos translation is essential. Furthermore, our results suggest that the role of nos mRNA localization in translational activation is to overcome smg-mediated repression. Thus, smg action would establish the link between nos RNA localization and nos translation.

\section{Results}

To search for translational control elements in the nos mRNA we used a nos/bcd transgene similar to one described by Gavis and Lehmann (1992). In this transgene the nos 3' UTR, which includes a posterior mRNA localization signal (Gavis and Lehmann 1992), has been replaced by the bcd 3' UTR, which contains an anterior localization signal (Macdonald and Struhl 1988). Consequently, the hybrid transcript encoded by the transgene is mislocalized to the anterior pole of the embryo, and ectopic nos protein causes the embryos to display a bicaudal phenotype in which anterior structures are replaced by a mirror image duplication of posterior structures (Gavis and Lehmann 1992).

We inserted regions of the nos 3' UTR into the nos/ $b c d$ transgene to determine whether any would suppress the bicaudal phenotype, suggesting they might mediate translational repression. Insertion of the first 96 nucleotides (nucleotides 1-96) of the 874-nucleotide nos 3' UTR largely reversed the nos/bcd bicaudal phenotype, restoring the normal polarity along the anteroposterior axis of the embryos as well as the normal number of abdominal and thoracic segments. This derivative of the nos/bcd transgene retained some anterior nos activity, as the embryos displayed head defects (Table 1; see Fig. 4 for a comparable cuticular phenotype). As expected for an RNA regulatory element, insertion of an antisense copy of the same region (nucleotides 96-1) into the nos/ $b c d$ transgene had no effect on the bicaudal phenotype. Suppression of the nos/bcd bicaudal phenotype by the 96-nucleotide region did not involve changes in mRNA localization, as the transgenic mRNA remained localized to the anterior pole of embryos (data not shown). Instead, the 96-nucleotide element mediated translational repression, as demonstrated below.

To determine whether the 96-nucleotide region could inhibit expression of a completely heterologous gene, we
Table 1. Mapping translational repression elements in the nos 3' UTR

\begin{tabular}{lccl}
\hline Transgene $^{\mathrm{a}}$ & Insertion $^{\mathrm{b}}$ & $\begin{array}{c}\text { Translational } \\
\text { repression }^{\mathrm{c}}\end{array}$ & Phenotype $^{\mathrm{d}}$ \\
\hline nos/bcd & none & no & Bicaudal \\
& $96-1$ & no & Bicaudal \\
& $1-96$ & yes & Head defects \\
& $1-80$ & yes & Head defects \\
& $1-64$ & no & Bicaudal \\
& $1-48$ & no & Bicaudal \\
& $1-32$ & no & Bicaudal \\
& $1-16$ & no & Bicaudal \\
& $21-96$ & yes & Head defects \\
& $41-96$ & no & Bicaudal \\
nosHA/bcd & $61-96$ & no & Bicaudal \\
& none & no & Bicaudal \\
& $1-96$ & yes & Head defects \\
& $96-1$ & no & Bicaudal \\
torso & $21-80$ & yes & Head defects \\
& $96-1$ & no & Wild-type \\
& $1-96$ & yes & torso \\
\hline
\end{tabular}

${ }^{a}$ Transgenes are as described in Materials and Methods.

'Insertion refers to sequences from the nos 3' UTR, numbered as noted in the text. Insertion in each case was at the start of the 3' UTR.

${ }^{\mathrm{c}}$ Translational repression is demonstrated for the nosHA/bcd transgenes later in the paper (Fig. 1), and is inferred in other cases based on the phenotypes associated with the transgenes. ${ }^{\mathrm{d}}$ Phenotypes were scored in an otherwise wild-type background for the nos/bcd and nosHA/bcd transgenes, and in a torso ${ }^{-}$ background for the torso transgenes.

${ }^{\mathrm{e}}$ This transgene provided low-level tor activity to a tor ${ }^{-}$mutant at both the anterior and posterior of the embryo.

introduced sense or antisense copies into a torso (tor) transgene. The maternal tor transcript is distributed throughout the embryo and specifies the terminal-most anterior and posterior structures (Schüpbach and Wieschaus 1986; Sprenger et al. 1989). An antisense copy of the 96-nucleotide region had no effect on the ability of the tor transgene to rescue the defects of tor $^{-}$mutants (Table 1). In contrast, a tor transgene carrying a sense copy of the 96-nucleotide element provided only a lowlevel tor activity at both the anterior and posterior of the embryo. Thus, the 96-nucleotide region was sufficient to inhibit expression of a heterologous gene in the absence of other nos sequences, and was active at both poles of the embryo.

To define more precisely the nos 3' UTR sequences required to repress nos/bcd expression, truncated forms of the 96-nucleotide region were inserted into the nos/ $b c d$ transgene and tested for body patterning phenotypes (Table 1). The ability to suppress the bicaudal phenotype was not affected when either the first 20 or last $16 \mathrm{nu}$ cleotides were deleted, indicating that those regions were dispensable for the inhibitory effect of the element. In contrast, further deletions from either end led to a loss of bicaudal suppression. Thus, the inhibitory activity was contained within a 60-nucleotide fragment, nucleotides 21-80 of the nos 3' UTR. 


\section{Translational repression and $m R N A$ degradation}

The observed suppression of the nos/bcd bicaudal phenotype by the 96-nucleotide region could involve effects on mRNA synthesis, mRNA stability, protein synthesis, or some combination of these options. To distinguish among the possibilities, an assay was required to detect directly the nos protein made from the nos/bcd transgene. The nos/bcd transgene was modified by addition of two carboxy-terminal influenza hemagglutinin epitope tags, giving nosHA/bcd. The epitope tags decreased the mobility of the nos protein in SDS-polyacrylamide gels, allowing us to resolve the tagged and endogenous nos proteins, both of which can detected in Western blots probed with anti-nos sera (Fig. 1). Addition of the epitope tag did not impair detectably the nos activity encoded by the nosHA/bcd transgene; females expressing the transgene produced bicaudal embryos (Table 1). Just as for the nos/bcd transgene, insertion of nos 3' UTR nucleotides 1-96 into the nosHA/bcd transgene suppressed the bicaudal phenotype (Table 1). The same result was obtained by inserting nucleotdes 21-80, demonstrating that this minimal region was indeed sufficient to mediate suppression.

Preliminary experiments to monitor expression of the nos $H A / b c d$ transgene and its derivatives revealed that substantial changes in mRNA levels occurred during the first $3 \mathrm{hr}$ of embryogenesis. Consequently, all analyses were performed with staged samples, corresponding to embryos $0-1,1-2$, or $2-3 \mathrm{hr}$ after egglaying, to define accurately differences in mRNA and protein levels. The unmodified nosHA/bcd transgene-directed synthesis of nosHA protein that appeared at lower levels in the 0 - to 1-hr embryos, and at higher levels in the 1- to 2- and 2- to 3-hr embryos (Fig. 1, top). Parallel RNase protection assays showed that the nosHA/bcd mRNA was present at high levels initially, and detected somewhat less at 2-3 hr (Fig. 1, middle). A similar pattern was observed when the nosHA/bcd transgene was modified by insertion of an antisense copy of the 96-nucleotide region. In contrast, insertion of a sense copy of the entire 96-nucleotide region, or just nucleotides 21-80, eliminated detectable nosHA protein at all times, although substantial levels of the mRNAs were present at the onset of embryogenesis. Thus, the regulatory element within nucleotides 21-80 repressed nos expression at the level of translation. In addition, the presence of this regulatory element had a delayed effect on mRNA stability, as the nosHA/bcd mRNAs carrying the nucleotides 1-96 or 21-80 insertions decayed prematurely (Fig. 1, middle). We conclude that the initial function of the regulatory element was to repress translation, and subsequently it promoted degradation of the nos $\mathrm{HA} / \mathrm{bcd}$ transcript.

Note that endogenous nos mRNA is translated, although it contains the same sequences that mediate translational repression of the nosHA/bcd mRNA. As described below, our results suggest that the translation of endogenous nos mRNA requires an activation event that overcomes this repression.

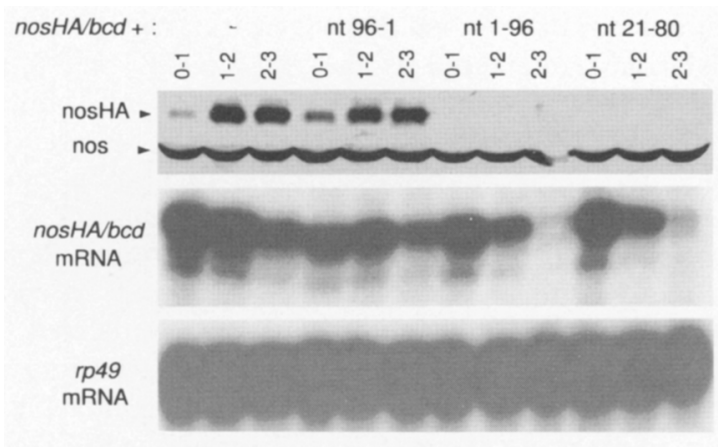

Figure 1. A region of the nos 3' UTR mediates translational repression. Samples used in each set of three lanes were from embryos of progressively more advanced stages $(0-1,1-2$, and 2-3 hr after egglaying). Transgenic females from which the embryos were obtained expressed the nosHA/bcd transgene $(-)$, or derivatives carrying insertions of the nos 3' UTR: nucleotides 1-96 in antisense (nucleotides 96-1) or sense (nucleotides 1-96) orientations, or nucleotides $21-80$ in the sense orientation (nucleotides 21-80). (Top) A Western blot probed with anti-nos serum showing time courses of the appearance of endogenous nos and transgenic nosHA proteins (the nos protein serves as a loading control). (Middle and bottom) RNase-protection assays monitoring the nosHA/bcd mRNA, as well as a control for RNA loading, the rp 49 mRNA. The presence of the sense version of either nucleotides $1-96$ or $21-80$ of the nos $3^{\prime}$ UTR eliminated detectable nosHA protein, and led to premature degradation of the encoding mRNA. Notably, when the transgenic nosHA protein did appear, it was always delayed relative to endogenous nos. We suspect that this reflects a form of translational control normally exerted on the $b c d$ mRNA; translation only begins after the poly(A) tail is extended shortly after egglaying (Sallés et al. 1994). Because cytoplasmic polyadenylation of bcd mRNA is controlled by the bcd mRNA 3' UTR (Sallés et al. 1994), which appears in the nosHA/bcd mRNA, extension of the poly(A) tail is presumably a prerequisite for translation of the nos $H A / b c d$ mRNA. In contrast, nos mRNA does not require poly(A) tail extension for translation, and appears to be translated from the onset of embryogenesis. We note that repression by $3^{\prime}$ UTR sequences can override activation conferred by cytoplasmic polyadenylation, if that is indeed the event responsible for the delay in nosHA translation. A similar effect is observed when the 96-nucleotide region of the nos mRNA 3' UTR is inserted into the tor mRNA, which is normally activated translationally by cytoplasmic polyadenylation (Sallés et al. 1994); repression still occurs (Table 1). It will be of interest to learn whether repression prevents cytoplasmic polyadenylation.

\section{A $135-k D$ protein binds specifically to the nos mRNA}

A UV cross-linking assay was used to test for the presence of proteins that interact specifically with nucleotides $21-80$ of the nos $3^{\prime}$ UTR, and potentially mediate translational repression. A number of proteins bound in a UV-dependent and extract-dependent manner to an RNA containing the 60-nucleotide region (Fig. 2, lane 1). However, competition binding experiments revealed that only one of these proteins bound specifically. Binding of a protein with an apparent molecular mass of 135$\mathrm{kD}$ was greatly reduced in the presence of excess specific 


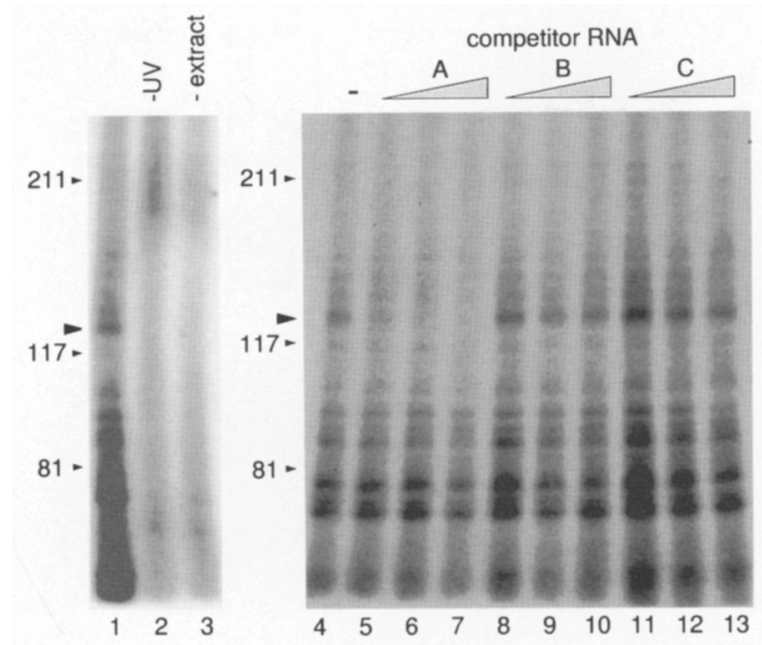

Figure 2. Protein binding to nucleotides $21-80$ of the nos mRNA 3' UTR. UV cross-linking assays were used to monitor binding of embryonic proteins to a labeled RNA containing nucleotides 21-80 of the nos 3' UTR. Results of typical assays (lanes 1,4). Most of the bands correspond to bound proteins, as demonstrated by their absence when the UV treatment (lane 2) or the protein extract (lane 3) is omitted from the assay. (Lanes 5-13). Competition binding experiments to demonstrate specificity of binding. Increasing amounts of unlabeled competitor RNAs $(10 \times, 30 \times$, and $100 \times$ molar excess over probe) were included in the binding reactions. (Lanes 5-7) A specific competitor; (lanes 8-10) BLE1 RNA competitor; and (lanes 11-13) BRE RNA competitor (see Materials and Methods). The binding of only one detectable protein of $\sim 135 \mathrm{kD}$ (indicated by the large arrowhead) was lost in the presence of the specific competitor but not in the presence of the nonspecific competitors. Note that neither the slight decrease in the $135-\mathrm{kD}$ protein in the presence of the nonspecific competitors or the apparent competition of other species at high concentration of specific competitor were reproducible. The positions at which molecular mass markers migrate are indicated.

competitor RNA (Fig. 2, lanes 5-7), but not in the presence of either of two nonspecific competitor RNAs (Fig. 2, lanes 8-13). No other protein displayed this behavior. We have designated the specifically bound protein smg. To determine when smg binding activity is present, extracts were prepared from different developmental stages. The binding activity was absent from ovary extracts, or extracts enriched for late stage eggs, and first appeared early in embryogenesis, peaked shortly thereafter, and was largely gone by $4 \mathrm{hr}$ after egg-laying (Fig. $3 \mid$.

\section{smg binding correlates with translational repression}

If smg mediates translational repression through the 60 nucleotide region, then its binding should correlate with the ability of the element to repress translation in vivo. This can be tested by studying the properties of point mutations in the 60-nucleotide region that do or do not affect binding. To identify the region of the 60-nucleotide to which smg binds, we first generated mutant RNAs bearing multiple random point mutations, and

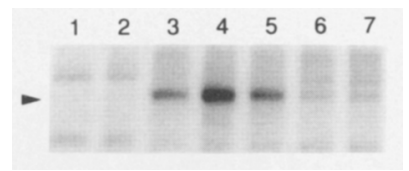

Figure 3. Time course of appearance of smg binding activity. Standard binding reactions were performed on staged extracts, using a labeled $3 \times$ SRE RNA probe (see Fig. 6) to enhance detection of smg. Extracts were prepared from whole ovaries (lane 1), late-stage oocytes (lane 2), and embryos aged 0-1, 1-2, 2-3, $3-4$, and 4-5 hr after fertilization (lanes 3-7, respectively). smg binding activity is indicated by an arrowhead.

tested them using the binding assay. Results from these experiments (data not shown) suggested that the smg binding site was located within nucleotides $25-40$ of the nos 3' UTR, a region that has the potential to form a stem-loop structure (Fig. 4A). Eight RNAs bearing single point mutations in the implicated region were tested for binding to smg. Four mutations blocked smg binding, as assayed by UV cross-linking, whereas four mutations did not (Fig. 4B).

Each point mutation was also tested for its effect on the ability of the 60-nucleotide region to repress translation of the nosHA/bcd mRNA. Each of the four mutants that retained smg binding suppressed the bicaudal phenotype, suggesting that they remained competent to mediate translational repression (Fig. 4E, F, H,I). In contrast, the four mutants unable to bind smg failed to suppress the bicaudal phenotype, suggesting that they were unable to mediate translational repression (Fig. 4G). To confirm that these body patterning phenotypes reflected effects on translation, protein and mRNA levels were tested for four selected mutations that did or did not prevent detectable smg binding (Fig. 5). The two point mutations competent for smg binding displayed the translational repression and mRNA degradation associated with the intact 60-nucleotide region, whereas the two point mutations that blocked smg binding were defective in both of these processes. These results suggest strongly that smg binding mediated translational repression and, perhaps indirectly, the subsequent mRNA degradation. We refer to the smg binding site as SRE. This site is referred to as SRE1, as a second SRE appears elsewhere in the nos mRNA (below).

Sequence inspection suggests that SRE1 is a stem-loop structure. Mutations that prevented smg binding are in the loop itself, or in the adjacent base pairs of the stem, showing that these regions were essential for binding. Mutations in the lower part of the predicted stem did not affect in vitro binding to smg, or the ability of the SRE to mediate substantial repression of translation. Nevertheless, for the latter mutations subtle changes in the extent of translational repression, as revealed by the extremely sensitive body patterning assay, were consistent with the folding of SRE1 into a stem-loop structure. Mutation $\mathrm{U} 42 \mathrm{C}$, which strengthened a potential weak G-U base pair, retained full SRE activity (Fig. 4I). Mutations A27U and $\mathrm{U} 41 \mathrm{~A}$, which both disrupted the same potential A-U 


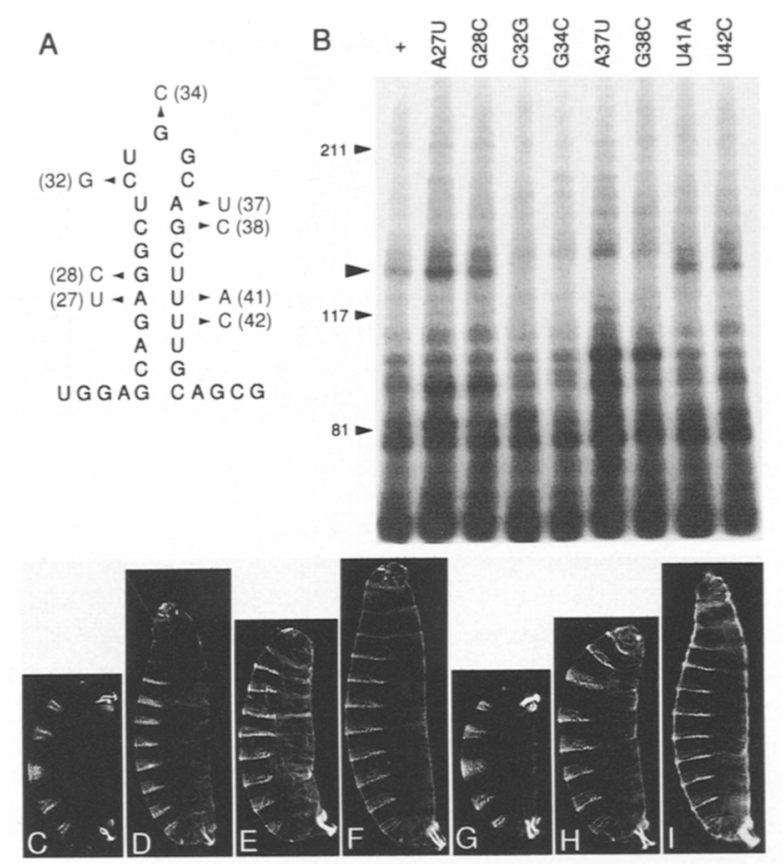

Figure 4. smg binding mediates suppression of the bicaudal phenotype by the SRE. $(A)$ Sequence of the SREl-containing region of the nos mRNA, drawn to indicate potential secondary structure. Point mutations used in the analysis are indicated, together with nucleotide position in the nos 3' UTR. (B) Binding assays using labeled point mutant RNAs as probes. The position of smg is indicated by a large arrowhead. Mutants C32G, G34C, $\mathrm{A} 37 \mathrm{U}$, and G38C did not bind smg detectably, whereas all other mutants bound smg much like the wild-type RNA $(+) .(C-I)$. Cuticular phenotypes of embryos obtained from mothers expressing nosHA/bcd transgenes. In $C$, the transgene lacked further nos 3' UTR sequences. In $D-I$ the transgenes carried insertions of nos 3' UTR nucleotides 21-80, either wild-type $(D)$ or bearing single point mutations: A27U $(E), \mathrm{G} 28 \mathrm{C}(F), \mathrm{C} 32 \mathrm{G}$, G34C, A37U, or G38C $(G)$; U41A $(H)$; U42C $(I)$. For mutations that prevented smg binding, the cuticular phenotypes $(G)$ were indistinguishable from those obtained when the transgene lacked nos 3' UTR nucleotides 21-80 (C). Mutations A27U and U41A, which affected two nucleotides that have the potential to base-pair, led to slightly increased levels of nos activity; the cuticles lacked thoracic segments $(E, H)$, which would otherwise be present $(D)$. Mutation U42C had no effect on the cuticular pattern $(I)$. Mutation G28C leads to mixed results. Some embryos had no change in the cuticular pattern $|F|$, whereas others lacked thoracic segments (data not shown).

base pair, displayed a modest reduction in SRE function. The level of ectopic nos activity produced by transgenes was now sufficient to interfere with thoracic segmentation (Fig. 4E and 4H, respectively), whereas the anterior nos activity present with the intact SRE only caused head defects (Fig. 4D). Finally, mutation G28C, which disrupted a potential weak G-U base pair, gave a mixture of embryos; some retained full SRE activity (Fig. 4F), whereas others had phenotypes similar to those of mutations A27U and U41A (data not shown). Thus, there is a correlation between the destabilization of the predicted stem and the strength of SRE activity.

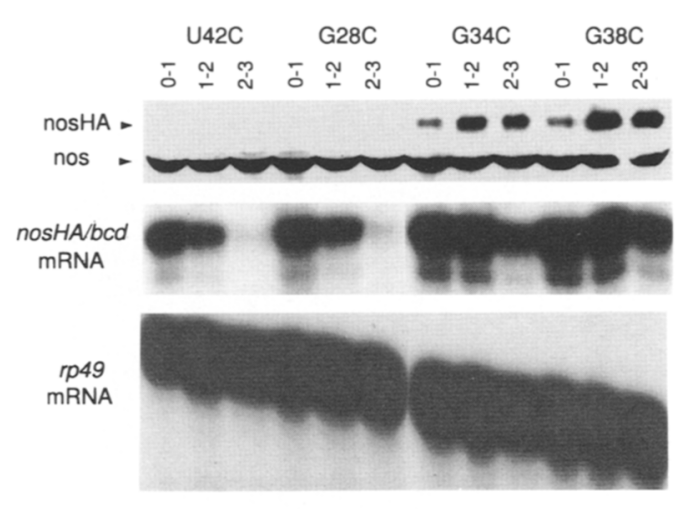

Figure 5. smg binding mediates translational repression by the SRE. Data on protein and mRNA levels are presented as in Fig. 1. Samples were from embryos of transgenic mothers expressing the nosHA/bcd gene with insertion of nucleotides 21-80 of the nos 3' UTR. The nucleotides 21-80 region carried the point mutations indicated above each set of lanes. Mutations U42C and G28C, which did not detectably impair smg binding in vitro, retained full SRE function; nosHA protein does not appear, and the encoding mRNAs are degraded prematurely. In contrast, mutations G34C and G38C, which failed to detectably bind smg, lacked all SRE activity (cf. Fig. 1).

Our analysis strongly suggests that binding of smg to the SRE is required for translational repression of the nosHA/bcd mRNA. Deletion analysis of the 96-nucleotide region of the nos $3^{\prime}$ UTR (Table 1) also indicated that the SRE-containing region was required, but identified additional essential sequences. For example, deletion of nucleotides 65-96, which leaves the SRE intact, also inactivated the element. It is possible that these deletions affected indirectly SRE function, perhaps through changes in RNA secondary structure; alternatively, the deleted region may contain the binding site for an additional factor required for translational repression. Given this ambiguity we wished to determine whether smg binding alone is sufficient to confer translational repression on the nosHA/bcd transgene. For these experiments we made use of an RNA containing three copies of the SRE $(3 \times$ SRE). One copy corresponded to SRE1, a second to SRE2 (see below), and a third SRE was synthetic, consisting of the loop sequence shared by SRE1 and SRE2, and a stem with novel sequences. A control RNA ( $\left.3 \times \mathrm{SRE}^{-}\right)$was identical, except for carrying mutations in each of the predicted loops. $3 \times \mathrm{SRE}$ RNA binds strongly to smg, whereas the $3 \times \mathrm{SRE}^{-}$RNA did not (Fig. 6A).

The in vivo activities of the $3 \times \mathrm{SRE}$ and $3 \times \mathrm{SRE}^{-}$ RNAs were monitored as described above using nosHA/ $b c d$ transgenes. The $3 \times$ SRE RNA supported full translational repression, as revealed indirectly in the body patterning assay (Fig. 6C) and directly by monitoring protein and RNA levels (Fig. 6B). In contrast, the $3 \times \mathrm{SRE}^{-}$ RNA was defective in translational repression (Fig. $6 \mathrm{~B}, \mathrm{D})$. These results strongly suggest that smg binding is sufficient to confer both translational repression and mRNA degradation to the nos $H A / b c d$ mRNA. 


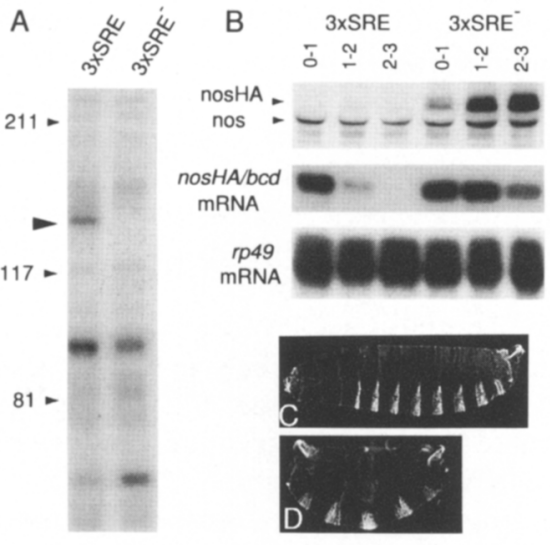

Figure 6. SREs are sufficient to confer translational repression on the nosHA/bcd mRNA. (A) Binding assays using labeled $3 \times$ SRE and $3 \times \mathrm{SRE}^{-}$RNAs as probes. smg, indicated by a large arrowhead, binds strongly to the $3 \times$ SRE RNA and not detectably to the $3 \times \mathrm{SRE}^{-}$. We confirmed that this protein was smg on the basis of its comigration with smg cross-linked to the original 60-nucleotide probe, and by competition binding experiments (data not shown). (B) Data on protein and mRNA levels are presented as in Fig. 1. Samples were from embryos of transgenic mothers expressing the nosHA/bcd gene with insertion of the $3 \times$ SRE or $3 \times \mathrm{SRE}^{-}$. The presence of the $3 \times$ SRE in the nosHA/bcd mRNA prevented translation of nosHA protein and led to premature degradation of the encoding mRNA, suppressing the bicaudal phenotype of the unmodified nos $H A / b c d$ transgene $(C)$. In contrast, inclusion of the $3 \times \mathrm{SRE}^{-}$affected neither translation nor mRNA stability, and failed to suppress the bicaudal phenotype $(D)$.

\section{SREs repress the translation of bona fide nos}

Our results have used artificial reporter genes to investigate the role of the SREs and smg in nos translation. A simple approach to testing the role of these components in regulation of endogenous nos is to create a nos transgene in which all smg binding sites have been mutated, and then monitor its properties in vivo. To perform such an experiment, we first searched for additional SREs in the nos 3' UTR. Competition binding experiments suggested that the nos 3' UTR contained one additional smg binding site located between nucleotides 97-185 (data not shown). Inspection of this sequence revealed a potential stem-loop structure (nucleotides 130-144) where the loop sequence CUGGC is identical to the loop in SRE1, whereas the stem sequence is different. To confirm that this second stem-loop, which we call SRE2, was a smg binding site, we introduced point mutations into the loops of SRE1, SRE2, or both, in the context of an RNA spanning nucleotides 1-185 of the nos 3' UTR. Both SRE1 and SRE2 must be mutated to eliminate binding of smg to this 185-nucleotide RNA (Fig. 7A). Thus, both sites bind smg, and there appears to be no other smg binding sites in the nos 3' UTR /or in the remainder of the nos mRNA, as judged by sequence inspection).

Both mutations were inserted into an otherwise wildtype nos transgene to make nosSRE ${ }^{-}$. Mothers expressing a wild-type nos transgene produced only wild-type

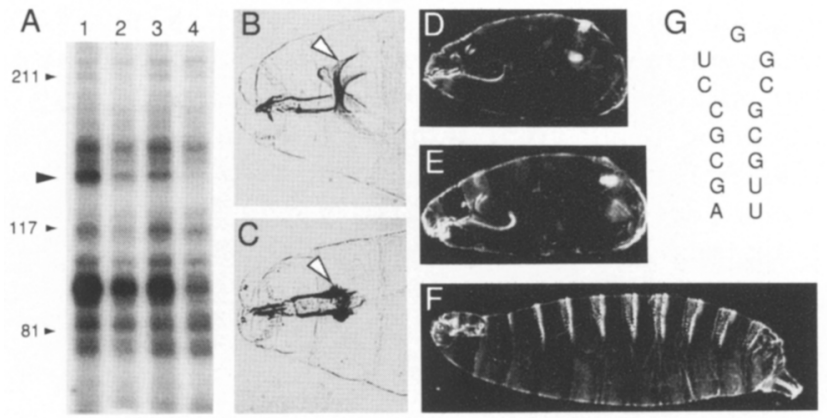

Figure 7. Action of the SREs in the nos mRNA. $(A)$ Binding assays using labeled RNA probes of wild-type nos 3' UTR nucleotides 1-185 (lane 1), or derivatives in which SRE1, SRE2, or both SRE1 and SRE2 were mutated (lanes 2-4, respectively). Wild-type SRE1 sequence CTGGCAG was mutated to CTGcagG. Wild-type SRE2 sequence CTGGCG was mutated to CTGcaG. When both SREs were mutated, smg binding was no longer detected. $(B, C)$ (phase contrast) Anterior cuticular regions of embryos from mothers expressing a wild-type nos transgene $(B)$ or the nosSRE $E^{-}$transgene $(C)$. The presence of the $n O S S R E^{-}$mRNA led to head defects (indicated by the reduced head skeleton; arrowheads). $(D-F)$ (dark field illumination) Localization of nos mRNA is dispensable in the absence of smgmediated translational repression. $(D)$ Cuticle from an embryo of an osk- mother. In the absence of osk activity, nos mRNA is neither localized nor translated, and the embryos lacked all abdominal structures. $(E)$ Cuticle from an embryo of an osk mother expressing a wild-type nos transgene. $(F)$ Cuticle from an embryo of an osk $^{-}$mother expressing a nosSRE $E^{-}$transgene. The abdominal segments formed normally, indicating that osk activity and hence, nos mRNA localization, were not required for nos translation or posterior body patterning if smg-mediated repression was eliminated. $(G)$ Sequence and predicted structure of SRE2.

embryos (Fig. 7B), wheres mothers expressing nosSREproduced embryos lacking normal head structures (Fig. 7C). This phenotype is consistent with overexpression of nos protein and demonstrates that SRE-mediated repression of nos expression is essential for normal development. This phenotype did not result from aberrant localization of the nOSSRE ${ }^{-}$mRNA as demonstrated by in situ hybridization (data not shown).

We then investigated the possibility that the SREs could mediate translational repression of nos mRNA that fails to localize to the posterior of the embryo. Such a model is consistent with previous work showing that nos mRNA is not translated if it is not localized to the posterior (Gavis and Lehmann 1994). To test this possibility, we assayed the levels of nos activity in an osk ${ }^{-}$ mutant carrying the nosSRE ${ }^{-}$transgene. Because osk is required to localize (and therefore translate) the nos mRNA (Ephrussi et al. 1991), osk ${ }^{-}$mutants lack nos activity, which is reflected by a lack of abdominal segmentation (Lehmann and Nüsslein-Volhard 1986) (Fig. 7D). However, the nosSRE ${ }^{-}$transgene rescued completely abdominal segmentation in an osk ${ }^{-}$mutant (Fig. $7 \mathrm{~F}$, note that the nosSRE ${ }^{-}$transgene still induces head defects in these embryos|, whereas a wild-type nos trans- 
gene did not (Fig. 7E). Thus, the activity of the nosSRE transgene in wild-type and an $o s k^{-}$mutant indicate that the phenotypic defects associated with the nOSSRE transgene result from translation of unlocalized nos mRNA. Consistent with these results, we detect a low uniform level of unlocalized nos protein in embryos carrying the nosSRE $E^{-}$transgene (data not shown).

\section{Discussion}

We have investigated the translational control of nos mRNA, a localized maternal transcript that directs posterior development (Wang and Lehmann 1991). We have characterized cis-acting elements SREs that mediated repression of translation, and have identified a protein, smg, that binds specifically to these sites. Mutations that eliminated smg binding also inactivated the function of the SREs, whereas mutations that retained smg binding retained substantial SRE function. These results suggest strongly that smg acted as a translational repressor. In our investigations we assayed SRE function in hybrid reporter genes and in the nos mRNA itself. The results from these approaches were both complementary and consistent. Each approach also provided additional information about nos translational control. For example, analysis of the hybrid reporter genes revealed that the SREs were active throughout the embryo. Also, a distinct activation event was required for posterior translation of nos mRNA (see below). Furthermore, the use of hybrid transgenes suggested that smg-mediated translational repression was associated with degradation of target mRNAs. Testing the role of SREs in their natural context, the nos mRNA allowed us to address the importance of translational repression to normal development; when the SREs were nonfunctional nos activity was no longer restricted to the posterior, despite normal localization of the nos mRNA. This ectopic nos expression resulted in lethal body patterning defects. These results suggest that mutants with altered regulation of nos mRNA translation will be defective in body patterning, facilitating a genetic analysis of this process.

\section{SREs mediate translational repression of unlocalized nos $m R N A$ in the embryo}

Expression of nos protein outside its normal posterior domain leads to body patterning defects (Ephrussi and Lehmann 1992; Gavis and Lehmann 1992; Smith et al. 1992). Such a situation might be prevented by translational repression at either of two different phases. First, repression could prevent accumulation of nos protein in the oocyte before posterior localization of nos mRNA, which occurs only late in oogenesis. Because nos protein cannot be detected in late-stage oocytes (Wang et al. 1994), nos mRNA must be repressed translationally or the nos protein must be unstable. In a similar situation earlier in oogenesis, translation of osk mRNA is repressed before its localization to the posterior pole of the oocyte (Kim-Ha et al. 1995). However, smg did not appear to suppress translation of nos mRNA before its localization within the oocyte, as we did not detect smg binding activity until early embryogenesis. There is some reason to suspect that protein instability may be responsible for the absence of nos protein in the oocyte. nos protein is present at high levels in late-stage nurse cells (Wang et al. 1994) that empty their contents into the oocyte. The failure to detect nos protein after this transfer suggests that it may be degraded upon entry into the oocyte. With such a protein degradation mechanism in place, there would be no need for translational repression during oogenesis.

A second opportunity for translational repression of nos mRNA occurs in the embryo. High levels of nos mRNA are localized to the posterior (Wang and Lehmann 1991), but substantial levels of the transcript are also distributed throughout the remainder of the embryo (our unpublished observations). Repression could limit the synthesis of nos protein from the unlocalized nos mRNA, and our results strongly suggest that this is the function of smg. When the SREs were mutated in an otherwise intact nos mRNA, nos activity was no longer limited to the posterior, as demonstrated by the anterior defects associated with the nOSSRE ${ }^{-}$transgene. Localization of the nosSRE ${ }^{-}$mRNA is not affected, but nos protein appears throughout the embryo, indicating that the unlocalized mRNA is being translated.

A role for smg in repressing unlocalized nos mRNA demands that smg activity be found throughout the embryo; our results using a tor transgene suggest that this is the case. tor activity normally appears at both poles of the embryo, and tor transgenes carrying an antisense (and therefore, nonfunctional) copy of an SRE provided complete tor function. However, tor transgenes carrying a sense copy of an SRE failed to provide tor activity at either end of the embryo, suggesting that smg is present at both locations. This result also bears on the posterior activation of nos translation, as discussed below.

Much of our analysis of the SREs was performed in the presence of additional nos mRNA sequences, which could also contribute to translational repression. This includes our experiment that used the tor transgene where the SRE was contained within a 96-nucleotide fragment of the nos 3' UTR. Thus, our data show that the SREs are necessary for repression of endogenous nos, but they do not reveal whether the normal complement of two SREs is sufficient for repression. The possibility that the nos message contains additional sequences that are required to repress nos translation will require further analysis of the nos transcript. However, our demonstration that insertion of a $3 \times$ SRE cassette into the nosHA/bcd transgene conferred translational repression does demonstrate that multiple SREs can function in the absence of other nos 3' UTR sequences.

All of the above data are consistent with the ability of the SREs to function independently and therefore, raise the possibility that SRE-like sequences may act to repress translation of other maternal mRNAs. However, our search of $\sim 45$ mRNAs that are expressed in the early 
embryo has not revealed any similar stem-loop structures (data not shown).

\section{Activation of nos translation}

Translation of nos mRNA is activated as a consequence of localization to the posterior pole of the embryo. In mutants defective in nos mRNA localization, such as osk $^{-}$, no nos protein can be detected (Gavis and Lehmann 1994). Given our model that smg represses translation throughout the embryo and that nos mRNA is normally only translated when localized to the posterior, it seems likely that the role of localization in nos translation is to overcome smg-mediated repression. If so, then localization should no longer be a prerequisite for nos translation if the SREs are mutated and nonfunctional. This is indeed the case, as osk ${ }^{-}$mutants carrying the nosSRE $E^{-}$transgene displayed normal abdominal segmentation, revealing the presence of substantial levels of nos activity. The failure to translate wild-type nos mRNA in an osk ${ }^{-}$mutant, whereas nosSRE $E^{-}$mRNA was translated, further strengthens our suggestion that smg serves to repress the translation of unlocalized nos mRNA (as detailed above).

How might localization overcome smg-dependent repression? Properties of the tor transgene carrying an SRE are instructive. The presence of the SRE led to loss of $t$ or activity at both poles of the embryo. The absence of posterior tor activity indicates that the SRE by itself did not mediate translational activation at the posterior pole. This result has two important implications. First, it shows that an additional cis-acting regulatory element is required for activation. Our preliminary work indicates that the nos 3' UTR does indeed contain sequences outside of nucleotides 1-96 (the site of SRE1) that mediate translational activation (C.A. Smibert and P.M. Macdonald, unpubl.). These results are consistent with work from Dahanukar and Wharton (this issue), who have shown that a segment of the nos mRNA encompassing SRE1 and SRE2 (nucleotides 1-184 of the 3' UTR) mediates both repression and activation of translation. Second, it indicates that the role of osk in activation cannot be limited to a direct effect on smg itself; if so, we would expect that the SRE/smg complex would be a suitable substrate for osk-mediated translational activation. This was not the case, as osk did not activate the expression of the posteriorly located tor mRNA-carrying SRE1.

The role of osk in posterior development likely involves the recruitment and maintenance of a ribonucleoprotein complex at the posterior that is responsible for posterior development (Ephrussi et al. 1991; Kim-Ha et al. 1991; Webster et al. 1994). Therefore, we speculate that osk recruits some factor to the posterior that overcomes smg-mediated repression, thus allowing for posterior-specific translation of nos mRNA. This model is consistent with the link between nos translation and nos RNA localization observed by Gavis and Lehmann (1994), although it invokes a different mechanism; they proposed that the act of localization itself activated translation. smg binding is tightly correlated with translational repression and $m R N A$ degradation

Our work with the nos/bcd hybrid transgenes suggests that translational repression occurred together with mRNA degradation. Time-course experiments show that repression precedes degradation. Two results imply that smg binding to SREs mediated both processes. First, SRE point mutations that prevented smg binding blocked both events. Second, hybrid transgenic mRNAs carrying the three tandem SREs were repressed translationally and degraded subsequently. These results join the growing body of evidence that translational control and RNA stability can be intimately linked (for review, see Theodorakis and Cleveland 1996). It is possible that smg -mediates both processes; alternatively, smg may repress specifically translation, and degradation occurs simply because the mRNA is not being translated.

Our results linking translational repression and mRNA degradation prompted us to re-examine the behavior of the endogenous nos mRNA. We found that substantial levels of unlocalized nos mRNA were present very early in embryogenesis, and that they were degraded before cellularization. If smg regulates the stability of this unlocalized nos mRNA, then we would expect unlocalized nosSRE ${ }^{-}$mRNA to persist longer than unlocalized wild-type nos mRNA. We found that, although the bulk of nOSSRE $E^{-}$was degraded like wild type, a small fraction of the nosSRE ${ }^{-}$mRNA persisted into cellularization (data not shown). These results suggest that more than one mechanism degrades nos mRNA. It would not be surprising if a more general system also destabilizes nos transcripts, as many maternal mRNAs are degraded rapidly early in embryogenesis.

\section{Why is nos expression so carefully regulated?}

One intriguing aspect of our results is that the elaborate system, involving at least 10 gene products, for localizing molecules to the posterior of the embryo (St. Johnston and Nüsslein-Volhard 1992) becomes dispensable for posterior body patterning if nos translational repression is alleviated by mutations in the SREs. Specifically, osk ${ }^{-}$mutants are completely defective in the posterior localization of a number of molecules, including the nos gene products, and lack any trace of posterior body patterning activity (Lehmann and Nüsslein-Volhard 1986; Hay et al. 1990; Lasko and Ashburner 1990; Raff et al. 1990; Jongens et al. 1992; Wang et al. 1994). However, complete posterior body patterning was restored in the presence of the nosSRE ${ }^{-}$transgene, which allowed translation of unlocalized nos mRNA. The only negative effect associated with this promiscuous translation was the disruption of head formation. These head defects resulted from the nos-dependent translational repression of the bcd mRNA, which is mediated by nos response elements (NREs) in the bcd mRNA 3' UTR (Wharton and Struhl 1991). We have found that such defects can be eliminated completely in animals expressing a form of the $b c d$ mRNA from which the NREs has 
been deleted (Webster et al. 1994). Thus, it would seem that the requirements for nos mRNA localization and translational repression are imposed by the presence of the NRE in the bcd mRNA.

\section{Materials and methods}

\section{Extract preparation}

All extracts were prepared from $w^{1118}$ animals. Embryos $(0-$ to l-hr after egglaying unless otherwise indicated) collected on yeasted apple juice plates were dechorionated in bleach for 1 min, washed extensively with $0.1 \%$ Triton X-100, and transferred to a $1.5-\mathrm{ml}$ microfuge tube (this and all subsequent steps were performed on ice or at $4^{\circ} \mathrm{C}$ ). The volume of the embryos was estimated and an equal volume of lysis buffer [50 mM Tris $\mathrm{HCl}(\mathrm{pH} 7.5), 20 \mathrm{~mm} \mathrm{NaCl}, 0.1 \%$ Triton X-100, $0.5 \mathrm{~mm}$ EDTA, $1 \mathrm{~mm}$ DTT, $1 \mathrm{~mm}$ PMSF, $2 \mathrm{~mm}$ benzamidine, $2 \mu \mathrm{g} / \mathrm{ml}$ of leupeptin, and $2 \mu \mathrm{g} / \mathrm{ml}$ of pepstatin] was added. Embryos were homogenized with a plastic pestle and centrifuged in a refrigerated microfuge at $15,000 \mathrm{rpm}$ for $15 \mathrm{~min}$. Glycerol was then added to the supernatant to a final concentration of $10 \%$, and extracts were stored at $-70^{\circ} \mathrm{C}$. Ovaries from well-fed 3- to 4-day-old females were hand-dissected into ice cold $0.1 \%$ Triton $\mathrm{X}-100$ and extracts were prepared as above. To prepare extracts enriched for late-stage eggs, well-fed 4-day-old virgin females were used (Wyman 1979); the enrichment was verified by visual inspection.

\section{UV cross-linking assay}

Probe synthesis and UV cross-linking assays were performed as described by Macdonald et al. (1995) with the following modifications: 3-6 $\mu \mathrm{L}$ of protein extract were combined in a $10-\mu \mathrm{L}$ reaction with $20 \mu \mathrm{g}$ of yeast tRNA, $1 \mu \mathrm{L}$ of $60 \mathrm{~mm}$ HEPES-OH (pH 7.9), $20 \mathrm{mM} \mathrm{MgCl}_{2}$, and $3 \times 10^{6} \mathrm{cpm}$ of ${ }^{32} \mathrm{P}$-labeled RNA. RNase digestion was done at room temperature using $50 \mu \mathrm{g}$ of RNase. Proteins were resolved on a $6 \%$ SDS-polyacrylamide gel. Probes were synthesized in vitro using either SP6 or T7 RNA polymerase (New England Biolabs) as per the manufacturer's instructions, using plasmid subclones as templates. For the nos 3' UTR nucleotides 21-80 probe, the subclone contained that region of nos DNA inserted in the BglII and BamHI sites of pSP72 (Promega). To estimate the position of the smg binding site, this DNA was amplified by error-prone PCR /Cadwell and Joyce 1992), and a collection of subclones were sequenced. Fourteen subclones, each bearing 1- to 5-point mutations /covering 22 of the 60 nucleotides of the probe), were used for probe synthesis and binding reactions. Directed point mutations were introduced by PCR into the wild-type DNA. Competition binding was performed as described (Macdonald et al. 1995). The specific competitor RNA was simply the probe RNA prepared without incorporation of the radioactive label. The nonspecific competitor RNAs carried either one copy of the bcd RNA localization signal BLEl (Macdonald et al. 1995) or four tandem bruno binding sites (Kim-Ha et al. 1995). Templates for the $3 \times$ SRE and $3 \times$ SRE $^{-}$RNAs were constructed by assembly of multiple oligonucleotides into the BglII and SalI sites of pSP73 (Promega). The $3 \times$ SRE sequence was 5'-GATCTGCAGAGGCTCTGGCAGCTTTTGCCCCGTAAGCGCCTTGGCGCGTTTCTAGAAATAATAATCAGCTGGCCTGIATTAG3 . The $3 \times \mathrm{SRE}^{-}$sequence differed in that each underlined $\mathrm{C}$ or $\mathrm{G}$ residue was converted to a $\mathrm{G}$ or $\mathrm{C}$, respectively. The template for synthesis of the RNA probe containing both SRE1 and SRE2 was a BamHI-BglII fragment corresponding to nucle- otides $1-185$ of the nos 3' UTR inserted into pGEM2 (Promega). Point mutations in the SREs were introduced by PCR.

\section{Transgenic flies}

Transgenic flies were generated by P-element-mediated transformation using $w^{1118}$ embryos as recipients (Spradling and $\mathrm{Ru}$ bin 1982). The nos/bcd transgene used in these experiments consisted of three genomic DNA segments, all incorporated into the CaSpeR transformation vector (Pirotta 1988): (1) a nos DNA fragment including $1.2 \mathrm{~kb}$ of 5 ' flanking sequences and the entire nos open reading frame, with a $\mathrm{BamHI}$ site introduced by PCR immediately after the stop codon; (2) a 0.6-kb EcoRV-StuI (sites converted to BamHI and SalI, respectively) fragment of the bcd 3' UTR that contains all of the bcd RNA localization signals but lacks the $b c d$ polyadenylation signal; and (3) $3.5-\mathrm{kb}$ of 3' flanking sequences from the nos gene beginning at an XhoI site immediately $5^{\prime}$ to the polyadenylation signal. The nosHA/ $b c d$ transgene differed only by addition at the end of the nos coding region of a synthetic DNA encoding two copies of the hemagglutinin epitope tag (Kolodziej and Young 1991). Additional DNA fragments from the nos 3' UTR were inserted into both constructs at the unique BamHI site separating the nos coding sequence and the bcd 3' UTR sequence. The nos 3' UTR fragments (all identified by sequence coordinates in the nos $3^{\prime}$ UTR) were prepared by PCR, and incorporated 5' BamHI and 3' $B g l I I$ sites to facilitate cloning. The wild-type nos and nosSRE $E^{-}$ transgenes consisted of the same nos $5^{\prime}$ and coding sequences described for nos/bcd, joined to a 4.2-kb nos genomic fragment beginning with a BamHI restriction site introduced by PCR immediately after the stop codon. For the nosSRE $E^{-}$transgene, PCR was used to introduce the nucleotide changes in the SRE sequences noted in the legend to Figure 7 . The tor transgene was as described previously (Wharton and Struhl 1991) and nucleotides 1-96 of the nos 3' UTR flanked by an XbaI site on one side and an SpeI site on the other were inserted into the SpeI insertion site in the tor $3^{\prime}$ UTR. Transgenic lines were used in the $w^{1118}$ background. For testing in mutant backgrounds, transgenic lines on the appropriate chromosome were identified by segregation tests, doubly balanced, and crossed into the appropriate background. For analysis in osk ${ }^{-}$mutants, flies were transheterozygous for $o s k^{54}$ and $o s k^{166}$. For analysis in tor $^{-} \mathrm{mu}-$ tants, flies were homozygous for tor $^{P M}$.

\section{RNA and protein analysis}

Embryo extracts for Western blot analysis were prepared as for UV cross-linking assays with the exception that embryos were homogenized in $2 \times$ protein sample buffer plus $1 \mathrm{mM}$ PMSF and the extracts were not centrifuged. After boiling for $5 \mathrm{~min}$, proteins were resolved on $10 \%$ SDS polyacrylamide gels and then transferred to nitrocellulose using a Genie Blotter (Idea Scientific) as per the manufacturer's instructions. nos proteins were detected using rat anti-nos antisera [provided by Robin Wharton [Duke University Medical Center, Durham, NC)] and the Western Light protein detection kit (Tropix). RNase protection was performed as described previously (Macdonald and Struhl 1988), using the same $r p 49$ probe. For detection of nosHA/bcd mRNA a probe corresponding to the $3^{\prime}$ end of the nosHA/bcd open reading frame (including the epitope tags) was generated to differentiate endogenous nos RNA from nosHA/bcd mRNA.

\section{Acknowledgments}

We thank Adam Harris, Yung Lie, Helen McNeill, and Philippa 
Webster for discussions and criticism of the manuscript and Robin Wharton for antibodies, fly stocks, and discussions about similar work in his laboratory. This work was supported by a David and Lucile Packard Fellowship and National Institutes of Health grant GM54409 to P.M.M. C.A.S. is a Terry Fox Research Fellow supported with funds provided by the Terry Fox Run. J.E.W. was supported by a National Science Foundation predoctoral fellowship.

The publication costs of this article were defrayed in part by payment of page charges. This article must therefore be hereby marked "advertisement" in accordance with 18 USC section 1734 solely to indicate this fact.

\section{References}

Cadwell, R.C. and G.F. Joyce. 1992. Randomization of genes by PCR mutagenesis. PCR Methods Appl. 2: 28-33.

Curtis, D., R. Lehmann, and P.D. Zamore. 1995. Translational regulation in development. Cell 81: 171-178.

Dahanukar, A. and R.P. Wharton. 1996. The Nanos gradient in Drosophila is generated by translational regulation. Genes \& Dev. (this issue).

Davidson, E.H. 1986. Gene activity in early development. Academic Press, Orlando, FL.

Ephrussi, A. and R. Lehmann. 1992. Induction of germ cell formation by oskar. Nature 358: 387-392.

Ephrussi, A., L.K. Dickinson, and R. Lehmann. 1991. oskar organizes the germ plasm and directs localization of the posterior determinant nanos. Cell 66: 37-50.

Gavis, E.R. and R. Lehmann. 1992. Localization of nanos RNA controls embryonic polarity. Cell 71: 301-313.

- 1994. Translational regulation of nanos by RNA localization. Nature 369: 315-318.

Hay, B., L.Y. Jan, and Y.N. Jan. 1990. Localization of vasa, a component of Drosophila polar granules, in maternal-effect mutants that alter embryonic anteroposterior polarity. Development 109: 425-433.

Jongens, T.A., B. Hay, L.Y. Jan, and Y.N. Jan. 1992. The germ cell-less gene product: A posteriorly localized component necessary for germ cell development in Drosophila. Cell 70: $569-584$.

Kim-Ha, J., J.L. Smith, and P.M. Macdonald. 1991. oskar mRNA is localized to the posterior pole of the Drosophila ooctye. Cell 66: 23-35.

Kim-Ha, J., K. Kerr, and P.M. Macdonald. 1995. Translational regulation of oskar mRNA by bruno, an ovarian RNA-binding protein, is essential. Cell 81: 403-412.

Kolodziej, P.A. and R.A. Young. 1991. Epitope tagging and protein surveillance. Methods Enzymol. 194: 508-519.

Lasko, P.F. and M. Ashburner. 1990. Posterior localization of vasa protein correlates with, but is not sufficient for, pole cell development. Genes \& Dev. 4: 905-921.

Lehmann, R. and C. Nüsslein-Volhard. 1986. Abdominal segmentation, pole cell formation, and embryonic polarity require the localized activity of oskar, a maternal gene in Drosophila. Cell 47: 141-152.

Macdonald, P.M. and G. Struhl. 1988. cis-acting sequences responsible for anterior localization of bicoid mRNA in Drosophila embryos. Nature 336: 595-598.

Macdonald, P.M., A. Leask, and K. Kerr. 1995. exl protein specifically binds BLE1, a bicoid mRNA localization element, and is required for one phase of its activity. Proc. Natl. Acac. Sci. 92: 10787-10791.

Markussen, F.-H., A.-M. Michon, W. Breitwieser, and A. Ephrussi. 1995. Translational control of oskar generates
Short OSK, the isoform that induces pole plasm assembly. Development 121: 3723-3732.

Pirotta, V. 1988. Vectors for P-mediated transformation in Drosophila. In Vectors: A survey of molecular cloning vectors and their uses (ed. R.L. Rodriguez and D.T. Denhardt), pp. 437-456. Butterworths, Boston, MA.

Raff, J.W., W.G.F. Whitfield, and D.M. Glover. 1990. Two distinct mechanisms localise cyclin B transcripts in syncytial Drosophila embryos. Development 110: 1249-1261.

Rongo, C., E.R. Gavis, and R. Lehmann. 1995. Localization of oskar RNA regulates oskar translation and requires Oskar protein. Development 121: 2737-2746.

Sallés, F.J., M.E. Lieberfarb, C. Wreden, J.P. Gergen, and S. Strickland. 1994. Coordinate initiation of Drosophila development by regulated polyadenylation of maternal messenger RNAs. Science 266: 1996-1999.

Schüpbach, T. and E. Wieschaus. 1986. Maternal-effect mutations altering the anterior-posterior pattern of the Drosophila embryo. Roux's Arch. Dev. Biol. 195: 302-317.

Smith, J.L., J.E. Wilson, and P.M. Macdonald. 1992. Overexpression of oskar directs ectopic activaton of nanos and presumptive pole cell formation in Drosophila embryos. Cell 70: 849-859.

Spradling, A.C. and G.M. Rubin. 1982. Transposition of cloned $\mathrm{P}$ elements into Drosophila germ line chromosomes. Science 218: 341-347.

Sprenger, F., L.M. Stevens, and C. Nüsslein-Volhard. 1989. The Drosophila gene torso encodes a putative receptor tyrosine kinase. Nature 338: 478-483.

St. Johnston, D. and C. Nüsslein-Volhard. 1992. The origin of pattern and polarity in the Drosophila embryo. Cell 68: 201219.

Theodorakis, N.G. and D.W Cleveland. 1996. Translationally coupled degradation of mRNA in eukaryotes. In Translation control. (ed. J.W.B. Hershey, M.B. Mathews, and N. Sonenberg), pp. 631-652. Cold Spring Harbor Laboratory Press, Cold Spring Harbor, NY.

Wang, C. and R. Lehmann. 1991. Nanos is the localized posterior determinant in Drosophila. Cell 66: 637-647.

Wang, C., L.K. Dickinson, and R. Lehmann. 1994. Genetics of nanos localization in Drosophila. Dev. Dynamics 199: 103115.

Webster, P.J., J. Suen, and P.M. Macdonald. 1994. Drosophila virilis oskar transgenes direct body patterning but not pole cell formation or maintenance of mRNA localization in $D$. melanogaster. Development 120: 2027-2037.

Wharton, R.P. and G. Struhl. 1991. RNA regulatory elements mediate control of Drosophila body pattern by the posterior morphogen nanos. Cell 67: 955-967.

Wyman, R. 1979. The temporal stability of the Drosophila oocyte. J. Embryol. Exp. Morph. 50: 137-144. 


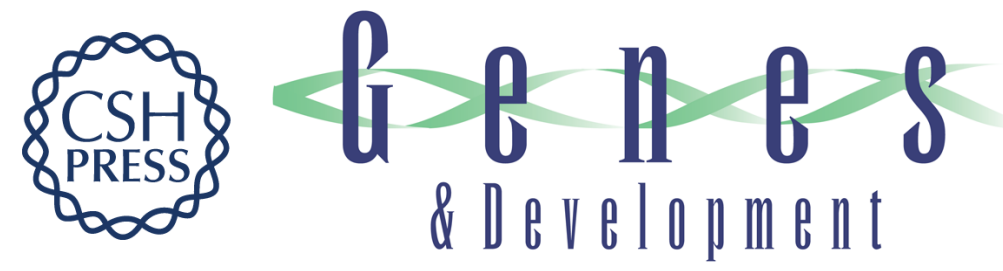

\title{
smaug protein represses translation of unlocalized nanos mRNA in the Drosophila embryo.
}

\author{
C A Smibert, J E Wilson, K Kerr, et al.
}

Genes Dev. 1996, 10:

Access the most recent version at doi:10.1101/gad.10.20.2600

References

This article cites 29 articles, 9 of which can be accessed free at: http://genesdev.cshlp.org/content/10/20/2600.full.html\#ref-list-1

\section{License}

Email Alerting

Receive free email alerts when new articles cite this article - sign up in the box at the top Service right corner of the article or click here.

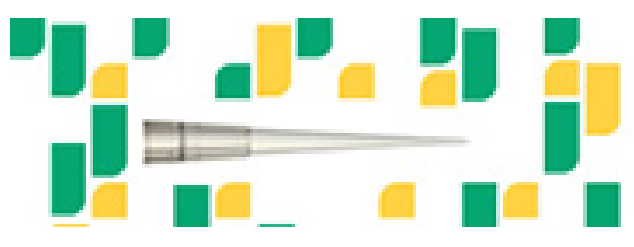

Focused on your science. 